19

\title{
Плазмон-поляритоны Дьяконова, распространяющиеся вдоль поверхности гиперболического метаматериала
}

\author{
(C) М.В. Давидович \\ Национальный исследовательский Саратовский государственный университет им. Н.Г. Чернышевского, \\ 410012 Саратов, Россия \\ e-mail: kiselevvm21@gmail.com
}

Поступила в редакцию 15.11 .2019 г.

В окончательной редакции 29.11.2019 г.

Принята к публикации 16.12.2019 г.

Рассмотрены плазмон-поляритоны типа поверхностных волн Дьяконова вдоль плоской границы гиперболического метаматериала с произвольной ориентацией кристаллографической оси. Найдены условия существования быстрых, медленных, втекающих, вытекающих, прямых и обратных плазмон-поляритонов. Рассмотрен волновод в виде асимметричного слоя гиперболического метаматериала. Приведено выражение для плотности электромагнитной энергии в таком метаматериале.

Ключевые слова: плазмон-поляритоны, плазмоны Дьяконова, гиперболический метаматериал, фотонный кристалл, дисперсионное уравнение, гомогенизация.

DOI: 10.21883/OS.2020.04.49207.311-19

\section{Введение}

В работе М.И. Дьяконова [1] рассмотрены поверхностные плазмон-поляритоны (ПП) вдоль границы одноосного кристалла и диэлектрика с диэлектрической проницаемостью (ДП) $\varepsilon$, когда оптическая ось параллельна плоскости раздела. Показано, что в случае $\varepsilon_{\|}>\varepsilon>\varepsilon_{\perp}$ возможно существование поверхностных волн, экспоненциально затухающих от границы и распространяющихся в некотором интервале углов относительно оси. Такие же волны исследованы в работе [2] на границе двух одноосных кристаллов, когда оси параллельны границе раздела и образуют между собой некоторый угол. При этом возникла необходимость представления поля через обыкновенную и необыкновенную волны. В работе [3] рассмотрены волны вдоль границы двух одинаковых анизотропных сред, в общем случае двуосных и немагнитных. Граница раздела получена поворотом двух полупространств с этими средами в разные стороны относительно нормали к ней. В работах [4-8] рассмотрен диэлектрический волновод (ДВ), стенки которого выполнены из недиссипативного гиперболического метаматериала (ГММ). ГММ представляют собой периодические искусственные среды, в которых возможно выполнение условия $\varepsilon_{\|}^{\prime} \varepsilon_{\perp}^{\prime}<0$, где штрих означает реальные части, а мнимые части весьма малы. Обычно их полагают равными нулю. Обзоры по ГММ даны в ряде работ [9-12]. Интересны ПП вдоль поверхностей асимметричных ГММ, т. е. прорезанных под углом к оптической оси $[13,14]$. В последнее время интерес к исследованию ПП на границах периодических метаматериалов, а также вдоль метаповерхностей $[15,16]$ возрос в связи с технологическими возможностями их изготовления и практическими потребностями их использования.
Цель данной работы - рассмотреть диссипативные ПП на границе ГММ, включая случай асимметричного ГММ. В упомянутых выше работах диссипация не учитывалась, искались именно волны, локализованные у поверхности. Однако только такими волнами не исчерпывается спектр волн: при диссипации кроме медленных поверхностных возможны быстрые поверхностные втекающие волны, а из слоя конечной толщины $t$ возможно излучение и быстрых вытекающих волн [16,17]. Все такие комплексные волны также будем называть ПП. В запрещенных зонах для поверхностных ПП (ППП) даже при отсутствии диссипации существуют решения в виде комплексных волн с комплексной (продольной) постоянной распространения и комплексными поперечными ее компонентами, а диссипация способствует переходу от одной ветви к другой [17]. ППП существуют за счет полного внутреннего отражения (ПВО). ПП возможны и при нарушении полного внутреннего отражения (НПВО), в частности за счет потерь. Ее энергия втекает в среду и диссипируется в ней [18]. Такая квазисобственная волна превращается в собственную при исчезновении потерь и восстановлении ПВО. Хороший пример - волна Ценнека, превращающаяся в волну, падающую под углом Брюстера при исчезновении потерь [18]. В ДВ (например, выполненных в виде слоя) возможны вытекающие антиповерхностные ПП (АПП) [18]. В такой волне НПВО происходит при уменьшении частоты ниже критической (на критической частоте фазовая скорость волны равна скорости света в вакууме), даже если нет диссипации, и в ней волна экспоненциально нарастает при удалении от поверхности. Такое нарастание есть следствие экспоненциального убывания волны при движении вдоль слоя [18]. АПП возможны в структурах, имеющих конечные (не нуле- 
вые и не бесконечные) поперечные сечения слоев. Из листа нулевой толщины (например, из листа графена) невозможно вытекание (если только он не активный, т.е. оптически накачанный), поскольку нет запасенной энергии. В такой диссипативный лист волна может только втекать из вакуума (с обеих сторон). Из диссипативного полупространства невозможно вытекание в вакуум, поскольку при этом волна будет бесконечно нарастать вглубь диссипативной среды и выделять бесконечную мощность. Такая волна есть волна, втекающая из вакуума в полупространство $[17,18]$ и затухающая в обе стороны от границы. Это означает соответствующий выбор ветви корней для нормальных компонент $k_{0 x}=\sqrt{k_{0}^{2}-k_{z}^{2}}$ в вакууме и $k_{x}=\sqrt{k_{0}^{2} \varepsilon-k_{z}^{2}}$ в среде. Это ППП, описываемый дисперсионным уравнением (ДУ) Ценнека $k_{z}=k_{0} \sqrt{\varepsilon /(\varepsilon+1)}$ (J. Zenneck, 1907), который всегда втекающий и может быть как быстрым, так и медленным (граница перехода от медленного ППП к быстрому лежит в области $-1<\varepsilon^{\prime}<0$ ). Таким образом, при учете диссипации есть медленные и быстрые втекающие ПП, а также быстрые вытекающие ПП.

Если рассматривать недиссипативные полупространства с ДП $\varepsilon$, то вытекающие в них волны можно рассматривать как быстрые по отношению в скорости $c / \sqrt{\varepsilon}$, т. е. такая волна может с одной стороны втекать из вакуума и быть медленной, а с другой стороны вытекать в среду, если ее фазовая скорость удовлетворяет условию $c / \sqrt{\varepsilon}<v_{p}<c$. Подводя к такому волноводу призму с ДП $\varepsilon$, можно ее использовать для вывода (или ввода) энергии. Вместо призмы можно использовать достаточно широкий ДВ со скошенным торцом, при этом ось ДВ должна быть направлена под углом вытекания. Таким образом, спектр комплексных ПП $F(\omega, \mathbf{k})=0$ состоит из ветвей, не имеющих разрывов в комплексном пространстве $\mathbf{k}=\mathbf{k}^{\prime}-i \mathbf{k}^{\prime \prime}$, а при разрешении этого ДУ в виде $\mathbf{k}=\mathbf{k}^{\prime}-i \mathbf{k}^{\prime \prime}=\mathbf{f}(\omega)$ ветви $\mathbf{k}^{\prime}=\mathbf{f}^{\prime}(\omega)$ и $\mathbf{k}^{\prime \prime}=\mathbf{f}^{\prime \prime}(\omega)$ не имеют разрывов в частотной области [17]. В бесконечном недиссипативном фотонном кристалле (ФК) разрешенное относительно частоты $\omega=\Omega(\mathbf{k})$ ДУ есть уравнение Френеля, которое при постоянстве частоты определяет поверхность изочастот в $\mathbf{k - п р о с т р а н с т в е ~ ( з д е с ь ~ в а ж н о , ~ ч т о ~} \mathbf{k}=\mathbf{k}^{\prime}$, т.е. это действительное трехмерное пространство). В случае диссипации $\mathbf{k}$-пространство шестимерное. В таком рассмотрении направление движения фазы $\boldsymbol{v}_{p}=\mathbf{k} /\left|\mathbf{k}^{\prime}\right|$, направление движения энергии $\boldsymbol{v}_{e}=\mathbf{S} /|\mathbf{S}|$ и направление движения диссипации $\boldsymbol{v}_{d}=\mathbf{k}^{\prime \prime} /\left|\mathbf{k}^{\prime \prime}\right|$ в общем случае не совпадают. Без диссипации можно ввести вектор групповой скорости $\mathbf{v}_{g}=\nabla_{\mathbf{k}} \omega=\left.\nabla_{\mathbf{k}} \Omega(\mathbf{k})\right|_{\mathbf{k}=\mathbf{k}^{\prime}}$. При этом возможны разрывы на кривых $\mathbf{k}^{\prime}=\mathbf{f}^{\prime}(\omega)$ в областях, в которых вектор $\mathbf{v}_{g}$ не определен. Его использование проблематично не только в этих областях уже при малой диссипации, но и в других областях при существенной диссипации.

Будем рассматривать слоистые одноосные ФК, называемые ГММ. При приведении к главной оси (ось направлена по оси $z$ ) в ГММ имеем $\varepsilon_{\perp}=\varepsilon_{x x}=\varepsilon_{y y}$, $\varepsilon_{\|}=\varepsilon_{z z}, \quad \varepsilon_{\perp}^{\prime} \varepsilon_{\|}^{\prime}<0$. Здесь штрих означает реальную часть. Для простоты будем рассматривать границу ГММ-вакуум, а решения уравнений максвелла искать в виде

$$
\begin{aligned}
\mathbf{E}(x, z, t) & =\mathbf{E} \exp \left(i\left(\omega t-k_{x} x-k_{y} y-k_{z} z\right)\right), \\
\mathbf{H}(x, z, t) & =\mathbf{H} \exp \left(i\left(\omega t-k_{x} x-k_{y} y-k_{z} z\right)\right) .
\end{aligned}
$$

Подставляя их в уравнения Максвелла, имеем соотношения для амплитуд

$$
\begin{aligned}
k_{z} H_{y}-k_{y} H_{z} & =\omega \varepsilon_{0} \varepsilon_{x x} E_{x}, \\
k_{x} H_{z}-k_{z} H_{x} & =\omega \varepsilon_{0} \varepsilon_{x x} E_{y}, \\
k_{y} H_{x}-k_{x} H_{y} & =\omega \varepsilon_{0} \varepsilon_{z z} E_{z}, \\
k_{y} E_{z}-k_{z} E_{y} & =\omega \mu_{0} H_{x}, \\
k_{z} E_{x}-k_{x} E_{z} & =\omega \mu_{0} H_{y}, \\
k_{x} E_{y}-k_{y} E_{x} & =\omega \mu_{0} H_{z} .
\end{aligned}
$$

Систему уравнений (1) можно записать в свернутом виде

$$
\hat{k} \mathbf{H}=\omega \varepsilon_{0} \hat{\varepsilon} \mathbf{E}, \quad-\hat{k} \mathbf{E}=\omega \mu_{0} \hat{I} \mathbf{H},
$$

где

$$
\hat{k}=\left[\begin{array}{ccc}
0 & k_{z} & -k_{y} \\
-k_{z} & 0 & k_{x} \\
k_{y} & -k_{x} & 0
\end{array}\right] .
$$

Такая запись верна для любого (в том числе и недиагонального) тензора $\hat{\varepsilon}$, для которого система (1) несколько усложняется, а также и для магнитного ФК при замене единичного тензора $\hat{I} \rightarrow \hat{\mu}$. Для вакуума $\hat{\varepsilon}=\hat{\mu}=\hat{I}$. Как нетрудно проверить, введенная матрица (2) особенная и обладает следующими свойствами: матрица $\hat{k}^{2}$ не особенная и $\hat{k} \hat{\varepsilon}=-(\hat{\varepsilon} \hat{k})^{T}$. Это справедливо для любой симметричной матрицы $\hat{\varepsilon}$. В случае диссипации у такой матрицы $\varepsilon_{m n}=\varepsilon_{n m}$, т.е. должны быть симметричными действительные и мнимые части ее элементов. В этом общем случае кристалла с диэлектрическими $\hat{\varepsilon}$ и магнитными $\hat{\mu}$ свойствами и в частном случае (1) равенство нулю определителя шестого порядка есть необходимое условие для существования искомого однородного решения. Поскольку матрица $\hat{k}$ особенная, каждое из уравнений можно разрешить единственным образом, например, $\mathbf{H}=-\left(\omega \mu_{0}\right)^{-1} \hat{k} \mathbf{E}$. При подстановке в первое уравнение получаем однородную систему трех уравнений в векторной форме

$$
-\hat{k}^{2} \mathbf{E}=k_{0}^{2} \hat{\varepsilon} \mathbf{E}
$$

и уравнение Френеля в виде

$$
\operatorname{det}\left(k_{0}^{2} \hat{\varepsilon}-\hat{k}^{2}\right)=0 .
$$

Этот определитель третьего порядка, однако в него входят квадраты компонент. Максимальная степень у 
компонент $\mathbf{k}$ четвертая, а у волнового числа $k_{0}$ может быть шестой. Из другого уравнения следует

$$
\mathbf{E}=\left(\omega \varepsilon_{0}\right)^{-1} \hat{\varepsilon}^{-1} \hat{k} \mathbf{H},
$$

система однородных уравнений $\left(k_{0}^{2} \hat{I}-\hat{k} \hat{\varepsilon}^{-1} \hat{k}\right) \mathbf{H}=0$ и уравнение Френеля

$$
\operatorname{det}\left(k_{0}^{2} \hat{I}-\hat{k} \hat{\varepsilon}^{-1} \hat{k}\right) \mathbf{H}=0 .
$$

Используя коммутационные свойства и симметричность тензора ДП, видим, что оба уравнения Френеля совпадают. Это имеет место и в более общем случае магнитодиэлектрического ФК, а также и для бианизотропного ФК. Для одноосного ФК уравнения Френеля разбиваются на два [19]: для обыкновенной волны $k_{z}^{2}=k_{0}^{2} \varepsilon_{\perp}$ и для необыкновенной волны $\varepsilon_{\|}^{-1}\left(k_{x}^{2}+k_{y}^{2}\right)+\varepsilon_{\perp}^{-1} k_{z}^{2}=k_{0}^{2}$. ПП с постоянной распространения $k_{z}= \pm k_{0} \sqrt{\varepsilon_{\perp}} \sqrt{1-\left(k_{x}^{2}+k_{y}^{2}\right) / \varepsilon_{\|}}$являются объемными $[16,20]$.

В качестве примера определения направления движения энергии рассмотрим объемный ПП в ФК, описываемом тензорами ДП $\hat{\varepsilon}$ и магнитной проницаемости $\hat{\mu}$. Пишем

$$
\begin{aligned}
& \mathbf{E}=\left(\omega \varepsilon_{0}\right)^{-1} \hat{\varepsilon}^{-1} \hat{k} \mathbf{H}=-\left(\omega \varepsilon_{0}\right)^{-1} \hat{\varepsilon}^{-1} \mathbf{k} \times \mathbf{H}, \\
& \mathbf{H}=-\left(\omega \mu_{0}\right)^{-1} \hat{\mu}^{-1} \hat{k} \mathbf{E}=\left(\omega \mu_{0}\right)^{-1} \hat{\mu}^{-1} \mathbf{k} \times \mathbf{E} .
\end{aligned}
$$

Для вектора Пойнтинга $\mathbf{S}=\operatorname{Re}\left(\mathbf{E} \times \mathbf{H}^{*}\right) / 2$ имеем

$$
\mathbf{S}=-\left(\omega \varepsilon_{0}\right)^{-1} \operatorname{Re}\left(\hat{\varepsilon}^{-1}\left(\mathbf{k} H^{2}-\mathbf{H}^{*} H_{k}\right)\right) / 2,
$$

или

$$
\mathbf{S}=c \mu_{0} \operatorname{Re}\left(\hat{\varepsilon}^{-1}\left(\mathbf{n} H^{2}-\mathbf{H}^{*} H_{n}\right)\right) / 2 .
$$

Здесь $\mathbf{n}=\mathbf{k} / k_{0}-$ комплексный вектор замедления (векторный индекс рефракции), $H^{2}=|\mathbf{H}|^{2}, H_{k}=\mathbf{k H}$. Далее все комплексные величины представляем в виде $\mathbf{n}=\mathbf{n}^{\prime}-i \mathbf{n}^{\prime \prime}$, где штрихом обозначена реальная часть, а двойным штрихом - отрицательная мнимая часть, векторными индексами обозначаем проекции. Имеем,

$$
\begin{gathered}
\mathbf{H}^{*} H_{\mathbf{n}}=\mathbf{H}^{\prime}\left(H_{\mathbf{n}^{\prime}}^{\prime}+H_{\mathbf{n}^{\prime}}^{\prime \prime}\right)-\mathbf{H}^{\prime \prime}\left(H_{\mathbf{n}^{\prime}}^{\prime \prime}-H_{\mathbf{n}^{\prime}}^{\prime}\right) \\
+i\left[\mathbf{H}^{\prime \prime}\left(H_{\mathbf{n}^{\prime}}^{\prime}+H_{\mathbf{n}^{\prime}}^{\prime \prime}\right)+\mathbf{H}^{\prime}\left(H_{\mathbf{n}^{\prime}}^{\prime \prime}+H_{\mathbf{n}^{\prime}}^{\prime}\right)\right], \\
\mathbf{S}=\frac{c \mu_{0}}{2}\left[( \hat { \varepsilon } ^ { - 1 } ) ^ { \prime } \left(\mathbf{n}^{\prime} H^{2}-\mathbf{H}^{\prime}\left(H_{\mathbf{n}^{\prime}}^{\prime}+H_{\mathbf{n}^{\prime \prime}}^{\prime \prime}\right)+\mathbf{H}^{\prime \prime}\left(H_{\mathbf{n}^{\prime}}^{\prime \prime}-H_{\mathbf{n}^{\prime \prime}}^{\prime}\right)\right.\right. \\
\left.\left.-\left(\hat{\varepsilon}^{-1}\right)^{\prime \prime}\left(\mathbf{n}^{\prime \prime} H^{2}+\left[\mathbf{H}^{\prime \prime}\left(H_{\mathbf{n}^{\prime}}^{\prime}+H_{\mathbf{n}^{\prime}}^{\prime \prime}\right)+\mathbf{H}^{\prime}\left(H_{\mathbf{n}^{\prime}}\right)^{\prime \prime}-H_{\mathbf{n}^{\prime}}^{\prime \prime}\right)\right]\right)\right] .
\end{gathered}
$$

Здесь также обозначено $\left(\hat{\varepsilon}^{-1}\right)^{\prime}=\operatorname{Re}\left(\hat{\varepsilon}^{-1}\right), \quad\left(\hat{\varepsilon}^{-1}\right)^{\prime}=$ $=-\operatorname{Im}\left(\hat{\varepsilon}^{-1}\right)$. Полученное выражение для вектора Пойнтинга обозначим $\mathbf{S}_{h}$. C другой стороны, можно получить такой же вектор Пойнтинга $\mathbf{S}_{e}$, выраженный через поле $\mathbf{E}$. Его получим, заменяя $\mathbf{H} \rightarrow \mathbf{E}, \hat{\varepsilon}^{-1} \rightarrow \hat{\mu}^{-1}, \mu_{0} \rightarrow \varepsilon_{0}$. Очевидно, должно быть $\mathbf{S}_{e}=\mathbf{S}_{h}$. Для доказательства следует использовать соотношения $\mathbf{k}\left(\mathbf{E}^{*} \times \mathbf{H}\right)=c \varepsilon_{0} \mathbf{E}^{*} \hat{\varepsilon} \mathbf{E}$ и $\mathbf{k} \cdot\left(\mathbf{E} \times \mathbf{H}^{*}\right)=c \mu_{0} \mathbf{H}^{*} \hat{\mu} \mathbf{H}$, следующие изуравнений Максвелла, а также сопряженные им соотношения, следующие из сопряженных уравнений Максвелла. Имеем

$$
\mathbf{k S}=c\left(\varepsilon_{0} \mathbf{E}^{*} \hat{\varepsilon} \mathbf{E}+\mu_{0} \mathbf{H}^{*} \hat{\mu} \mathbf{H}\right) / 4 .
$$

При отсутствии диссипации сразу следует соотношение

$$
2 \mathbf{k S}=c \varepsilon \operatorname{Re}\left(\mathbf{E}^{*} \hat{\varepsilon} \mathbf{E}\right)=c \mu_{0} \operatorname{Re}\left(\mathbf{H}^{*} \hat{\mu} \mathbf{H}\right) .
$$

Указанные уравнения удобны как дополнительные условия, накладываемые на тензоры при гомогенизации. Указанная процедура необходима для получения свойств ФК. В электродинамике известна проблема определения плотности энергии, особенно при диссипации, поскольку конструкции типа $W_{E}=\left(\mathbf{E} \hat{\varepsilon}^{*} \mathbf{E}^{*}+\mathbf{E}^{*} \hat{\varepsilon} \mathbf{E}\right) / 4$ и $W_{H}=\left(\mathbf{H} \hat{\mu}^{*} \mathbf{H}^{*}+\mathbf{H}^{*} \hat{\mu} \mathbf{H}\right) / 4$ в общем случае ее не определяют [18]. Проблема связана с запасенной энергией в веществе $[18,19]$, требует нестационарного рассмотрения и в общем случае должна решаться с учетом всей предыстории процесса запаса энергии [21]. Поскольку перенос вещества в использованных балансных уравнениях не рассматривался, приведенные соотношения отвечают переносимой вдоль движения волны электромагнитной энергии, которая может быть и отрицательной. В случае соотношений типа (1) имеем

$$
\mathbf{S}_{e}=c \varepsilon_{0}\left(\mathbf{n}^{\prime} E^{2}-\operatorname{Re}\left(\mathbf{E}^{*}\left(\mathbf{n}^{*} \mathbf{E}\right)\right)\right) / 2 .
$$

Видим, что в общем случае направление вектора Пойнтинга не совпадает с направлением движения фазы $\mathbf{n}^{\prime}$, при этом, если $\mathbf{n}^{\prime} \cdot \mathbf{S}<0$, то волна обратная. Если совпадают направления $n^{\prime}$ и $\mathbf{n}^{\prime \prime}$, а все проекции поля $\mathbf{H}$ на это направления равны нулю, то

$$
\mathbf{S}_{h}=c \mu_{0} H^{2}\left[\left(\hat{\varepsilon}^{-1}\right)^{\prime} \mathbf{n}^{\prime}-\left(\hat{\varepsilon}^{-1}\right)^{\prime \prime} \mathbf{n}^{\prime}\right] / 2 .
$$

Однако это тривиальный случай изотропной среды. Для нее

$$
\mathbf{S}_{h}=c \mu_{0} H^{2}\left(\varepsilon^{\prime} \mathbf{n}^{\prime}+\varepsilon^{\prime \prime} \mathbf{n}^{\prime \prime}\right) /\left(2|\varepsilon|^{2}\right)
$$

и также

$$
\mathbf{S}_{e}=c \varepsilon_{0} E^{2}\left(\mu^{\prime} \mathbf{n}^{\prime}+\mu^{\prime \prime} \mathbf{n}^{\prime \prime}\right) /\left(2|\mu|^{2}\right) .
$$

При этом $\quad \mathbf{n}=\boldsymbol{v}_{0} \sqrt{\varepsilon \mu}, \quad \mathbf{H}=\sqrt{\mu_{0} \mu /\left(\varepsilon_{0} \varepsilon\right)} \mathbf{E}, \quad$ поэтому $\mathbf{S}_{h}=\mathbf{S}_{e}$. В изотропном случае имеем

$$
n=\sqrt{\left(\varepsilon^{\prime} \mu^{\prime}-\varepsilon^{\prime \prime} \mu^{\prime \prime}\right)-i\left(\varepsilon^{\prime} \mu^{\prime \prime}+\mu^{\prime} \varepsilon^{\prime \prime}\right)},
$$

$$
\begin{aligned}
& n^{\prime}= \\
& \pm \sqrt{\sqrt{\left(\varepsilon^{\prime} \mu^{\prime}-\varepsilon^{\prime \prime} \mu^{\prime \prime}\right)^{2}+\left(\varepsilon^{\prime} \mu^{\prime \prime}-\mu^{\prime} \varepsilon^{\prime \prime}\right)^{2}}+\left(\varepsilon^{\prime} \mu^{\prime}-\varepsilon^{\prime \prime} \mu^{\prime \prime}\right)}, \\
& n^{\prime \prime}= \\
& \pm \sqrt{\sqrt{\left(\varepsilon^{\prime} \mu^{\prime}-\varepsilon^{\prime \prime} \mu^{\prime \prime}\right)^{2}+\left(\varepsilon^{\prime} \mu^{\prime \prime}-\mu^{\prime} \varepsilon^{\prime \prime}\right)^{2}}-\left(\varepsilon^{\prime} \mu^{\prime}-\varepsilon^{\prime} \mu^{\prime \prime}\right)} .
\end{aligned}
$$


Знак в (3) определяется условием $n^{\prime \prime}>0$, и формально отрицательный знак возможен, только если одновременно $\varepsilon^{\prime}<0$ и $\mu^{\prime}<0$. Однако такие изотропные метаматериалы не достижимы, а условие $\mu^{\prime}<0$ невозможно и для пространственно однородных сред [22]. Следовательно, однородная плоская волна в изотропной однородной среде всегда прямая. Однако на границах сред возможны комбинации неоднородных плоских волн, при этом движение энергии пограничной волны вдоль заданного направления получается путем усреднения вектора Пойнтинга по поперечному сечению и направлениям $\mathbf{k}_{\perp}$. В этом случае вектор $\mathbf{k}_{\|}=\mathbf{k}_{z}$ задает это направление движения. Он может быть выбран вдоль любой из осей (взята ось $z$ ), а условием обратной волны является условие $k_{z}^{\prime} k_{z}^{\prime \prime}<0$ [17]. Оно говорит о том, что фаза и энергия движутся в разные стороны, а направление движения энергии совпадает с направлением вектора $\mathbf{k}^{\prime \prime}$, в направлении которого волна затухает.

\section{Постановка задачи}

Нас будут интересовать Е-волны относительно оси $z$ (рис. 1), идущие вдоль поверхностей $x=0$ и $x=t$, для которых $H_{z}=0$. Для Н-волн $E_{z}=0$, и если $E_{x} \neq 0$, то эта Н-волна относительно оси $z$ является также Е-волной относительно оси $y$, поэтому дисперсия для этой задачи получается из решения ДУ первой задачи путем замены $\varepsilon_{x x} \leftrightarrow \varepsilon_{z z}$. При этом $k_{z}=0$. Если же при этом выполнено $E_{x}=E_{z}=0$, то это Н-волна, ДУ которой весьма простое. В нашем случае имеем

$$
\begin{aligned}
& H_{x}=\frac{k_{y} E_{z}-k_{z} E_{y}}{\omega \mu_{0}}=\frac{k_{0} \varepsilon_{x x} k_{y} E_{z}-k_{x} k_{z} Z_{0} H_{z}}{Z_{0}\left(k_{0}^{2} \varepsilon_{x x}-k_{z}^{2}\right)}, \\
& H_{y}=\frac{k_{z} E_{x}-k_{x} E_{z}}{\omega \mu_{0}}=\frac{-Z_{0} k_{y} k_{z} H_{z}-k_{0} \varepsilon_{x x} k_{x} E_{z}}{Z_{0}\left(k_{0}^{2} \varepsilon_{x x}-k_{z}^{2}\right)}, \\
& E_{x}=\frac{-k_{y} H_{z}+k_{z} H_{y}}{\omega \varepsilon_{0} \varepsilon_{x x}}=\frac{-Z_{0} k_{0} k_{y} H_{z}-k_{x} k_{z} E_{z}}{k_{0}^{2} \varepsilon_{x x}-k_{z}^{2}}, \\
& E_{y}=\frac{-k_{z} H_{x}+k_{x} H_{z}}{\omega \varepsilon_{0} \varepsilon_{x x}}=\frac{-k_{y} k_{z} E_{z}+Z_{0} k_{0} k_{x} H_{z}}{k_{0}^{2} \varepsilon_{x x}-k_{z}^{2}},
\end{aligned}
$$

при этом все компоненты связаны ДУ Френеля для необыкновенной волны (для частного случая обыкновенной волны оно также дает решение). Здесь и далее

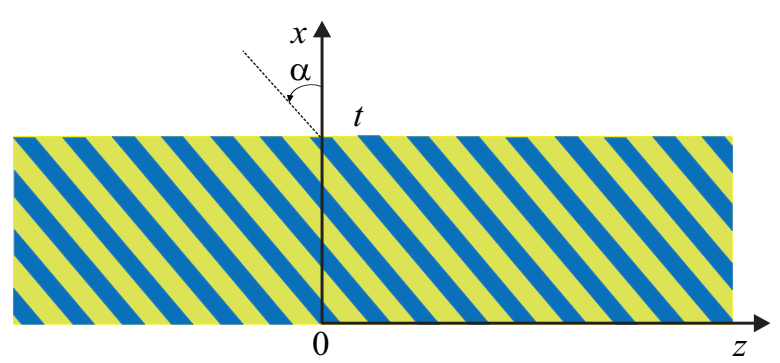

Рис. 1. Пластина ГММ толщины $t$ в виде слоев металл-диэлектрик с повернутой осью анизотропии.
$Z_{0}=\sqrt{\mu_{0} / \varepsilon_{0}}=c \mu_{0}$ означает характеристический импеданс вакуума. Полагаем $H_{z}=0$. Имеем связи

$$
\begin{aligned}
& E_{y}=E_{z} k_{y} k_{z} /\left(k_{z}^{2}-k_{0}^{2} \varepsilon_{x x}\right), \\
& E_{x}=E_{z} k_{x} k_{z} /\left(k_{z}^{2}-k_{0}^{2} \varepsilon_{x x}\right),
\end{aligned}
$$

а также связи в виде волновых сопротивлений

$$
\begin{gathered}
\frac{E_{z}}{H_{z}}=\frac{Z_{0}\left(k_{0}^{2} \varepsilon_{x x}-k_{z}^{2}\right)}{k_{y} k_{0} \varepsilon_{x x}}, \\
-\frac{E_{z}}{H_{y}}=\frac{Z_{0}\left(k_{0}^{2} \varepsilon_{x x}-k_{z}^{2}\right)}{k_{x} k_{0} \varepsilon_{x x}}, \\
-\frac{E_{y}}{H_{x}}=\frac{Z_{0} k_{z}}{k_{0} \varepsilon_{x x}} .
\end{gathered}
$$

Нас интересует ПП вдоль границы раздела $x=0$. Если рассмотреть в вакууме Е-волну, то для нее $k_{0}^{2}=k_{x 0}^{2}+k_{y}^{2}+k_{z}^{2}$. Первое и третье волновые сопротивления в (3) соответствуют переносу энергии вдоль границы, второе - в перпендикулярном к ней направлении. Сшиванию полей соответствует согласование волновых сопротивлений [17]:

$$
\frac{k_{0}^{2}-k_{z}^{2}}{k_{x 0}^{2}}=\frac{k_{0}^{2} \varepsilon_{x x}-l_{z}^{2}}{k_{x} \varepsilon_{x x}} .
$$

При этом достаточно использовать по одной волне в каждой среде. Равенство (4) и есть ДУ ПП вдоль поверхности. Оно может быть записано в виде

$$
\frac{k_{0}^{2}-k_{z}^{2}}{\sqrt{k_{0}^{2}-k_{y}^{2}-k_{z}^{2}}}=\frac{k_{0}^{2} \varepsilon_{x x}-k_{z}^{2}}{\varepsilon_{x x} \sqrt{k_{0}^{2} \varepsilon_{z z}-\varepsilon_{z z} / \varepsilon_{x x} k_{z}^{2}-k_{y}^{2}}} .
$$

Это биквадратное уравнение относительно $k_{z}$. При $k_{y}=0$ оно имеет простое решение

$$
k_{z}= \pm k_{0} \sqrt{\varepsilon_{x x}\left(\varepsilon_{z z}-1\right) /\left(\varepsilon_{x x} \varepsilon_{z z}-1\right)} .
$$

В случае однородной среды $\varepsilon_{x x}=\varepsilon_{z z}=\varepsilon$ оно принимает вид

$$
k_{z}= \pm k_{0} \sqrt{\varepsilon /(\varepsilon+1)},
$$

т.е. описывает втекающие поверхностные быстрые ПП (при $\operatorname{Re}(\sqrt{\varepsilon /(\varepsilon+1)})<1)$, а также медленные ППП (при $\operatorname{Re}(\sqrt{\varepsilon /(\varepsilon+1)})>1)$. В случае поворота границы раздела на угол $\pi / 2$ относительно оси $y$ в ДУ ПП (5) следует сделать замены $\varepsilon_{x x} \leftrightarrow \varepsilon_{z z}$ :

$$
k_{z}= \pm k_{0} \sqrt{\varepsilon_{z z}\left(\varepsilon_{x x}-1\right) /\left(\varepsilon_{x x} \varepsilon_{z z}-1\right)} .
$$

В этом случае ось перпендикулярна границе раздела, и все направления движения равноправны. Для ПП вдоль оси у также имеем

$$
k_{y}= \pm k_{0} \sqrt{\varepsilon_{z z}\left(\varepsilon_{x x}-1\right) /\left(\varepsilon_{x x} \varepsilon_{z z}-1\right)} .
$$

Гомогенизация периодической плоскослоистой среды из двух компонентов в виде слоя металла с ДП $\varepsilon_{m}$ и 
слоя диэлектрика с ДП $\varepsilon_{d}$ в приближении без учета пространственной дисперсии дает

$$
\varepsilon_{x x}=\varepsilon_{m} K_{m}+\varepsilon_{c} K_{d}, \quad \varepsilon_{z z}^{-1}=\left(\varepsilon_{m}^{-1} K_{m}+\varepsilon_{d}^{-1} K_{d}\right)^{-1} .
$$

Здесь ось ГММ направлена по оси $z$. В случае одинаковых коэффициентов заполнения $K_{m}=K_{d}=1 / 2$, $d_{m}=d_{d}=d / 2$, где $d-$ период, имеем

$$
\varepsilon_{x x}=\left(\varepsilon_{m}+\varepsilon_{d}\right) / 2, \varepsilon_{z z}=2 \varepsilon_{m} \varepsilon_{d} /\left(\varepsilon_{m}+\varepsilon_{d}\right) .
$$

ДП металла, оцениваемая по формуле Друде-Лоренца

$$
\varepsilon_{m}=\varepsilon_{L}-\omega_{p}^{2} /\left(\omega^{2}-i \omega \omega_{c}\right),
$$

в пренебрежении диссипацией можно считать отрицательной, если $\omega<\omega_{p} / \sqrt{\varepsilon_{L}}$. Тогда $\varepsilon_{x x}<0$, если $\varepsilon_{m}<-\varepsilon_{d}$. При этом $\varepsilon_{z z}>0$. Если же $-\varepsilon_{d}<\varepsilon_{m}<0$, то $\varepsilon_{x x}>0$ и $\varepsilon_{z z}<0$. Первый случай соответствует ГММ второго типа, а второй - ГММ первого типа. Отметим, что эти условия легко можно уточнить при учете диссипации, т. е. при конечном значении частоты столкновений $\omega_{c}$, при этом, конечно, следует говорить о значениях реальных частей $\varepsilon_{x x}^{\prime}$ и $\varepsilon_{z z}^{\prime}$. Гиперболическая дисперсия означает волны с высоким значением $|\mathbf{k}|=k \gg k_{0}$ (high$\mathrm{k}$ waves). Однако неограниченных значений быть не может. Имеет место ограничение $k \ll 2 \pi / d$, связанное с гомогенизацией, а также ограничение, связанное с диссипацией. Последнее при комнатной температуре более сильное. Оно может быть снижено использованием сверхнизких температур, и тогда при периоде в несколько нанометров можно ожидать достижение максимальных значений $k \sim 0.1 \mathrm{~nm}^{-1}$. Мы будем рассматривать асимметричный ГММ, ось которого повернута на угол $\alpha$. Рассмотрим матрицу поворота вокруг оси $y$ на угол $\alpha$ :

$$
\hat{T}(\alpha)=\left[\begin{array}{ccc}
\cos (\alpha & 0 & -\sin (\alpha) \\
0 & 1 & 0 \\
\sin (\alpha) & 0 & \cos (\alpha)
\end{array}\right]
$$

Действуя на вектор $\mathbf{E}$, она дает

$$
\begin{gathered}
E_{x}^{\prime}=E_{x} \cos (\alpha)-E_{z} \sin (\alpha), E_{y}^{\prime}=E_{y}, \\
E_{z}^{\prime}=E_{x} \sin (\alpha)+E_{z} \cos (\alpha),
\end{gathered}
$$

т.е. имеет место поворот против часовой стрелки. При этом исходная матрица ДП примет вид

$$
\tilde{\varepsilon}=\hat{T}^{-1}(\alpha) \hat{\varepsilon} \hat{T}(\alpha)=\hat{T}(-\alpha) \hat{\varepsilon} \hat{T}(\alpha)
$$

$$
\begin{aligned}
& \text { или } \\
& \tilde{\varepsilon}= \\
& =\left[\begin{array}{ccc}
\cos ^{2}(\alpha) \varepsilon_{x x}+\sin ^{2}(\alpha) \varepsilon_{z z} & 0 & \sin (\alpha) \cos (\alpha)\left(\varepsilon_{z z}-\varepsilon_{x x}\right) \\
0 & \varepsilon_{x x} & 0 \\
\sin (\alpha) \cos (\alpha)\left(\varepsilon_{z z}-\varepsilon_{x x}\right) & 0 & \cos ^{2}(\alpha) \varepsilon_{x x}+\sin ^{2}(\alpha) \varepsilon_{z z}
\end{array}\right]
\end{aligned}
$$

При таком повороте в соотношениях (1) следует учесть недиагональные компоненты тензора (7). В случае движения ПП вдоль оси $z$ имеем ДУ $\rho_{0}=\rho, \quad$ где $\rho_{0}=\sqrt{1-k_{z}^{2} / k_{0}^{2}}, \quad \rho=\sqrt{\left(\tilde{\varepsilon}_{x x}-k_{z}^{2} / k_{0}^{2}\right) / \Delta}$, $\Delta=\tilde{\varepsilon}_{x x} \tilde{\varepsilon}_{z z}-\tilde{\varepsilon}_{x z}^{2}$. Это уравнение также можно разрешить относительно $k_{z}$. Если рассмотреть задачу о слое несимметричного ГММ толщины $t$, то следует учесть волны двух направлений вдоль оси $x$, и тогда сшивание полей приводит к ДУ вида

$$
2 \rho \rho_{0} \alpha=\rho^{2}+\rho_{0}^{2},
$$

распадающегося на два:

$$
\rho_{0}=\rho\left(\alpha \pm \sqrt{\alpha^{2}-1}\right) .
$$

Здесь

при этом

$$
\alpha=\frac{\exp \left(-i k_{x}^{+} t\right)+\exp \left(-i k_{x}^{-} t\right)}{\exp \left(-i k_{x}^{+} t\right)-\exp \left(-i k_{x}^{-} t\right)}
$$

$$
k_{x}^{ \pm}=-k_{z} \tilde{\varepsilon}_{x z} / \tilde{\varepsilon}_{x x} \pm \sqrt{\left(k_{0}^{2} \tilde{\varepsilon}_{x x}-k_{z}^{2}\right) \Delta} / \tilde{\varepsilon}_{x x}
$$

В случае симметрии $\tilde{\varepsilon}_{x z}=0$, и ПП имеют либо электрическую, либо магнитную стенки в центре слоя.

Рассмотрим теперь ПП, движущийся под углом к оси $z$ вдоль границы такого асимметричного ГММ. Здесь уже нужно учесть две волны $E$ и $H$ вдоль оси $z$. Выражаем все поля в ГММ через $A E_{z}$ и $B H_{z}$ с произвольными амплитудами. В вакууме берем амплитуды $A_{0} E_{z}$ и $B_{0} H_{z}$. Сшиваем компоненты $E_{z}, H_{z}, E_{y}$, и $H_{y}$ получаем однородную систему линейных уравнений. При сшивании $E_{z}$ и $H_{z}$ сразу имеем $A-0=A, B_{0}=B$. Для поперечных компонент имеем

$$
\begin{gathered}
E_{x}=\frac{\left(k_{0}^{2} \tilde{\varepsilon}_{x z}+k_{z} k_{x}\right) E_{z}+\omega \mu_{0} k_{y} H_{z}}{k_{z}^{2}-k_{0}^{2} \tilde{\varepsilon}_{x x}}, \\
E_{y}=\frac{k_{z} k_{y} k_{x} E_{z}-\omega \mu_{0} k_{x} H_{z}}{k_{z}^{2}-k_{0}^{2} \tilde{\varepsilon}_{x x}}, \\
H_{x}=\frac{k_{z} k_{x} H_{z}-\omega \varepsilon_{0} \varepsilon_{x x} k_{y} E_{z}}{k_{z}^{2}-k_{0}^{2} \varepsilon_{x x}}, \\
H_{y}=\frac{k_{z} k_{y} H_{z}+\omega \varepsilon_{0}\left(\tilde{\varepsilon}_{x x} k_{x+} \tilde{\varepsilon}_{x z} k_{z}\right) E_{z}}{k_{z}^{2}-k_{0}^{2} \tilde{\varepsilon}_{x x}} .
\end{gathered}
$$

ДУ для такого ПП имеет вид

$$
\frac{\left(k_{z} k_{y}\right)^{2}}{k_{0}^{2}}=\frac{\left(\frac{k_{x}}{k_{z}^{2}-k_{0}^{2} \varepsilon_{x x}}-\frac{k_{x 0}}{k_{z}^{2}-k_{0}^{2}}\right)\left(\frac{\tilde{\varepsilon}_{x x} k_{x}+\tilde{\varepsilon}_{x z} k_{z}}{k_{z}^{2}-k_{0}^{2} \tilde{\varepsilon}_{x x}}-\frac{k_{x 0}}{k_{z}^{2}-k_{0}^{2}}\right)}{\left(\frac{1}{k_{z}^{2}-k_{0}^{2}}-\frac{1}{k_{z}^{2}-k_{0}^{2} \tilde{\varepsilon}_{x x}}\right)\left(\frac{1}{k_{z}^{2}-k_{0}^{2} \varepsilon_{x x}}-\frac{1}{k_{z}^{2}-k_{0}^{2}}\right)}
$$

При $k_{z}=0$ или $k_{y}=0$ левая часть обращается в нуль, и ДУ распадается на два. В последнем случае имеем ДУ

$$
k_{x} k_{x 0}=k_{0}^{2} \varepsilon_{x x}-k_{z}^{2} \text { и } k_{x 0}\left(\tilde{\varepsilon}_{x x} k_{x}+\tilde{\varepsilon}_{x z} k_{z}\right)=k_{0}^{2} \tilde{\varepsilon}_{x x}-k_{z}^{2} .
$$

В симметричном случае последнее уравнение приобретает вид $k_{x 0} k_{x}=k_{0}^{2}-k_{z}^{2} / \tilde{\varepsilon}_{x x}$. Симметричный случай возникает при $\alpha=0$ (для него $\tilde{\varepsilon}_{x x}=\varepsilon_{x x}$ ) и $\alpha=\pi / 2$ $\left(\tilde{\varepsilon}_{x x}=\varepsilon_{z z}\right)$,

$$
k_{z}^{2}-k_{0}^{2} k_{z}^{2}-k_{0}^{2} \tilde{\varepsilon}_{x x}=k_{0}^{2}-k_{z}^{2} / \tilde{\varepsilon}_{x x} .
$$




\section{Плотность энергии в ГММ}

Плотность энергии для дисперсии среды, описываемой ДП $\varepsilon_{m}(\omega)$ с законом Друде-Лоренца, известна. Считаем, что резонансные частоты в члене $\varepsilon_{L}(\omega)$ лежат существенно выше рассматриваемой частоты $\omega$ и почти не оказывают влияние, т. е. $\varepsilon_{L}(\omega)=\varepsilon_{L}=$ const. Поэтому плотность энергии в металле описывается выражением $W=W_{e}+W_{m}$, в котором

$$
\begin{gathered}
W_{E}=\varepsilon_{0}\left[\varepsilon_{L}+\omega_{p}^{2} /\left(\omega^{2}+\omega_{c}^{2}\right)\right] \mathbf{E}^{2} / 4, \\
W_{H}=\mu_{0} \mathbf{H}^{2} / 4 .
\end{gathered}
$$

Поскольку $\mathbf{H}=\sqrt{\varepsilon_{0}\left(\varepsilon_{m}^{\prime}-i \varepsilon_{m}^{\prime \prime}\right) / \mu_{0}} \mathbf{E}$, то для металла

$$
W_{m}=\varepsilon_{0}\left[2 \varepsilon_{m}^{\prime}+\sqrt{\varepsilon_{m}^{\prime 2}+\varepsilon_{m}^{\prime \prime 2}}\right] \mathbf{E}^{2} / 4
$$

При отсутствии диссипации имеем

$$
W_{m}=\varepsilon_{0}\left(\varepsilon_{L}+\left|\varepsilon_{m}^{\prime}\right|\right) \mathbf{E}^{2} / 2
$$

ниже частоты $\omega_{p} / \sqrt{\varepsilon}_{L}$, а выше нее

$$
W_{m}=\varepsilon_{0} \varepsilon_{L} \mathbf{E}^{2} / 2 \text {. }
$$

Считаем, что и диэлектрик описывается формулой Лоренца, все резонансные частоты которой также лежат далеко. Тогда $W_{d}=\varepsilon_{0} \varepsilon_{d} \mathbf{E}^{2} / 2$, и полная средняя за период плотность энергии в ГММ дается формулой

$$
W_{H M}=\left(W_{m} d_{m}+W_{d} d_{d}\right) / d
$$

Компонента вектора Пойнтинга $S_{x}$ показывает поток энергии в каждой из областей и определяет втекание/вытекание. Компоненту $S_{z}$ следует интегрировать по бесконечному поперечному сечению волноведущей структуры, как и плотность энергии для определения скорости ее переноса вдоль оси $z$. В случае поверхностной волны в пластине $|x|<t / 2$, затухающей по закону $\exp \left(-k_{0}^{\prime \prime}|x \mp t / 2|\right)$ вне пластины толщины $t$, величины $S_{z}$ и $W$ затухают по закону $\exp \left(-2 k_{0 x}^{\prime \prime}|x \mp t / 2|\right)$, поэтому интегралы существуют, а результат $v_{\mathrm{E}}=S_{z} / W$ дает скорость движения энергии. В случае вытекающей волны зависимость нарастающая $\exp \left(2 k_{0 x}^{\prime \prime}|x \mp t / 2|\right)$, и интегралы расходятся. Однако можно взять большую область $t / 2<|x|<L$, вычислить интегралы по ней и по области $|x|<t / 2$, вычислить $v_{\mathrm{E}}$ для нее и перейти к пределу $L \rightarrow \infty$. Получим конечный результат. Поскольку почти вся энергия переносится в вакууме, можно не интегрировать по пластине. Аналогичный результат дает усреднение по $L$. Перенос в вакууме идет под углом вытекания $\theta$ со скорость света. Поэтому вдоль пластины фаза движется со скоростью $c / \cos (\theta)$, а энергия со скоростью $c \cos (\theta)$, т. е. волна быстрая. Для ППП важен перенос энергии в пластине, от которого зависит тип волны: быстрая она или медленная, прямая или обратная.

\section{Численные результаты}

Мы рассматриваем металлические слои с параметрами $\varepsilon_{L}=9.0, \omega_{p}=1.6 \cdot 10^{6}, \omega_{c}=3.8 \cdot 10^{14} \mathrm{~Hz}$ и диэлектрические слои с $\varepsilon_{d}=3.0$. Для использования длин волн вплоть до $300 \mathrm{~nm}$ достаточно использовать период $d \sim 30 \mathrm{~nm}$. Слой (пластина) асимметричного ГММ представлен на рис. 1. Он является волноводом для ПП вдоль оси $z$. Результаты итерационного решения ДУ (8) приведены на рис. 2 для разных углов $\alpha$. Результаты расчета дисперсии и потерь для ПП вдоль границы полубесконечного образца ГММ при $\alpha=0$ и $\alpha=\pi / 2$ приведены на рис. 3 и 4. Часть кривых левее $n^{\prime}=1$ соответствует быстрым втекающим волнам, а правее медленным. Волны в плоскослоистой среде с $\alpha=\pi / 2$ более медленные, а в области выше частоты плазмонного резонанса обратные. Это отличает плоскослоистую среду от металлической, для которой обратных волн нет. Для ГММ с $\alpha=0$ обратных волн нет, а максимальное замедление соответствует более высоким частотам, для которых $\varepsilon_{m} />0$. Кривые построены так, что $k_{z}^{\prime}>0$, поэтому обратным волнам соответствуют как бы отрицательные потери. В вакууме энергия всегда переносится вдоль движения фазы. Наличие обратных волн - это интегральный эффект, связанный с тем, что в металлических структурах компонента вектора Пойнтинга может менять знак при $\varepsilon_{m}^{\prime}<0$.

В области плазмонного резонанса $k_{z}^{\prime} \approx k_{z}^{\prime} \sim 1 / \sqrt{\varepsilon^{\prime \prime}}$, т.е. для получения больших замедлений, следует снижать диссипацию. Оценка для первой структуры дает в

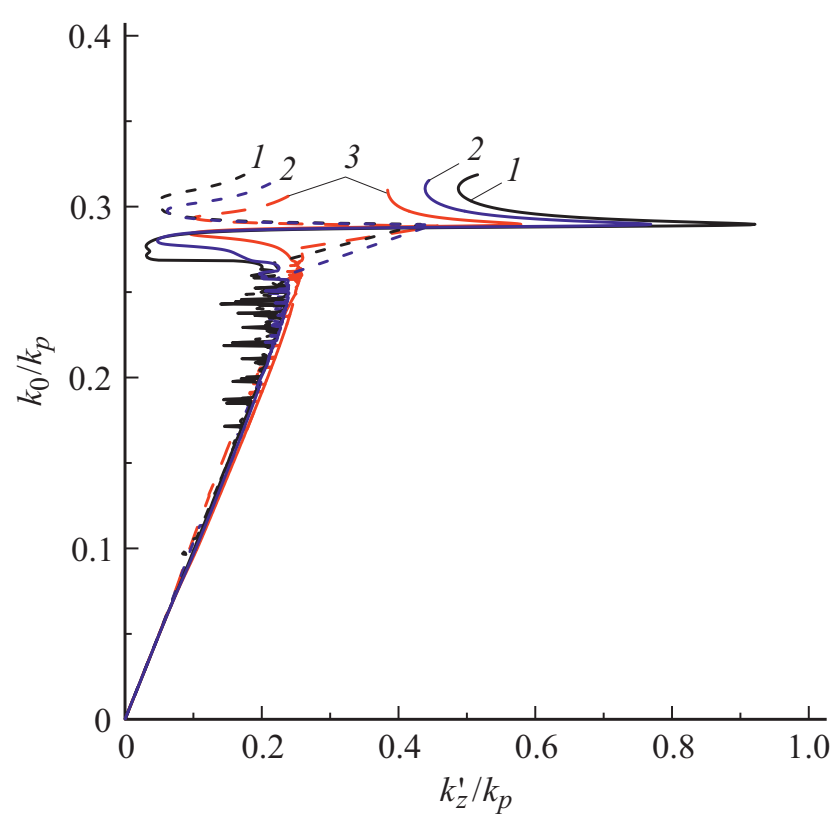

Рис. 2. Дисперсия в слое асимметричного ГММ толщины $50 \mathrm{~nm}$ при разных углах $\alpha: \pi / 3(1), \pi / 4$ (2) и $\pi / 6$ (3). Сплошные кривые соответствуют более медленным ПП по сравнению с штриховыми кривыми, что определяется двумя ветвями ДУ. 


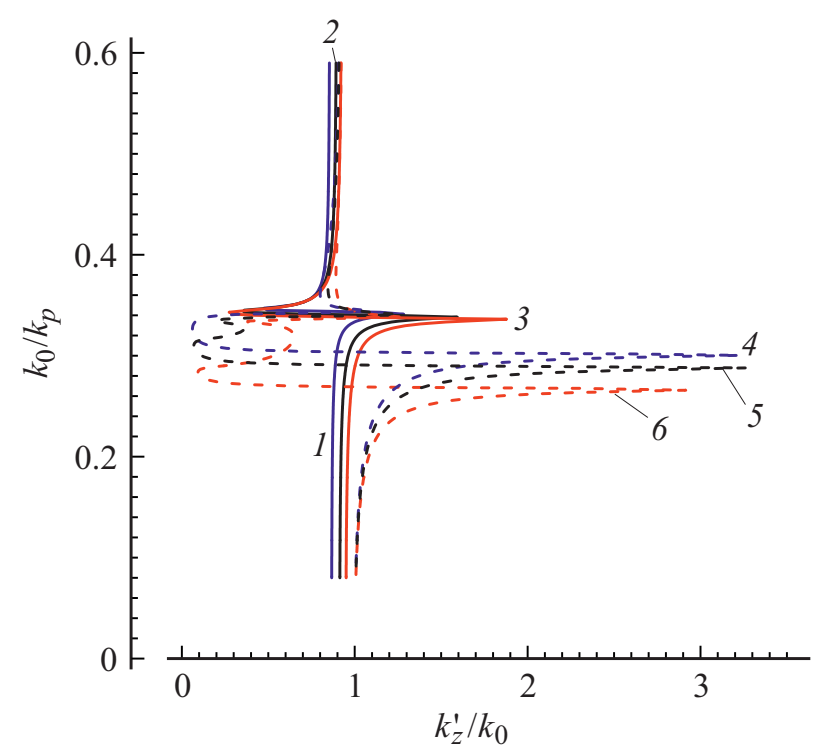

Рис. 3. Дисперсия Е-ПП вдоль полупространства ГММ со структурой, соответствующей $\alpha=0(1-3)$ и $\alpha=\pi / 2$ (4-6): зависимость нормированного волнового числа от замедления $n^{\prime}=k_{z}^{\prime} / k_{0}$ при разных ДП $\varepsilon_{d}: 2.0$ (синие кривые 1,4 ), 3.0 (черные 2,5 ) и 5.0 (красные 3,6 ).

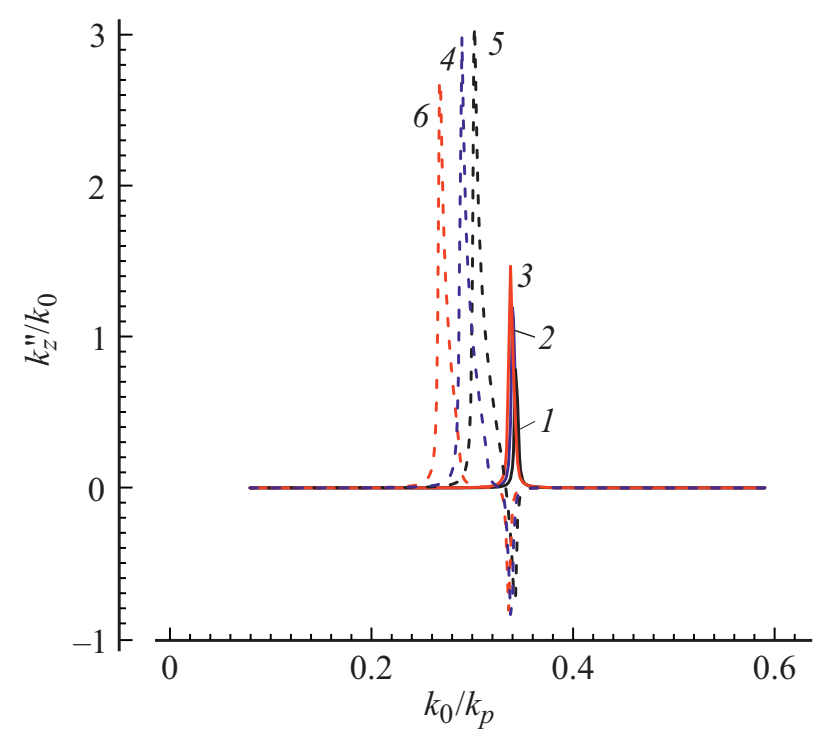

Рис. 4. Нормированные потери $k_{z}^{\prime} / k_{p}$ Е-ПП вдоль полупространства ГММ, соответствующие дисперсии рис. 2 для $\alpha=0(1-3)$ и $\alpha=\pi / 2(4-6)$ в зависимости от нормированного волнового числа $k_{0} / k_{p}$ при разных ДП $\varepsilon_{d}: 2.0$ (синие кривые 1,4), 3.0 (черные 2,5) и 5.0 (красные 3,6 ).

области резонанса

$$
k_{z}=k_{0}(1-i) \sqrt{\left(1+\varepsilon_{d}^{2}-2 / \varepsilon_{d}\right) / \varepsilon^{\prime \prime}} / 2 .
$$

Для второй структуры имеют место два резонанса

$$
k_{z}=k_{0}(1-i) \varepsilon_{d} / \sqrt{\left(1+\varepsilon_{d}^{2}\right) \varepsilon_{\prime \prime}^{\prime \prime}}
$$

и

$$
k_{z}=k_{0}(1+i) \sqrt{\left(1+\varepsilon_{d}^{2}-\varepsilon_{d}\right) /\left[\left(1+\varepsilon_{d}^{2}\right) 2 \varepsilon^{\prime \prime}\right]}
$$

- соответственно низкочастотный при $\varepsilon_{m} \approx-\varepsilon_{d}$ и высокочастотный при $\varepsilon_{m} \approx 1 / \varepsilon_{d}$. Непосредственно видно, что последний соответствует обратному ПП. Все исследованные ПП втекающие, поскольку вытекание из диссипативного полупространства невозможно.

\section{Заключение}

В работе с использованием простейшей гомогенизации получены точные решения для ПП Дьяконова вдоль поверхности в общем случае асимметричного гиперболического метаматериала в виде плоскослоистой периодической металл-диэлектрической структуры. Учет пространственной дисперсии, т.е. зависимости $\tilde{\varepsilon}\left(k_{0}, k_{x}, k_{z}\right)$, приводит к сложным нелинейным ДУ и уравнениям Френеля, анализировать которые возможно только численно. Как пространственная дисперсия, так и диссипация искажают гиперболический закон дисперсии и ограничивают по модулю компоненты волнового вектора, т.е. замыкают поверхность изочастот. Найдены условия существования медленных и быстрых, втекающих и вытекающих, а также прямых и обратных поляритонов. Рассмотрен волновод в виде слоя асимметричного гиперболического метаматериала. В качестве реализации ГММ в терагерцовом диапазоне удобно использовать структуры с листами графена. В таких ГММ возможна компенсация потерь при использовании лазерной оптической накачки в видимом диапазоне.

\section{Финансирование работы}

Работа была частично поддержана грантом РНФ проект № 16-19-10033.

\section{Конфликт интересов}

Автор заявляет, что у него нет конфликта интересов.

\section{Список литературы}

[1] Дьяконов М.И. // ЖЭТФ. 1988. Т. 94. № 4. С. 119; D'yakonov M.I. // Sov. Phys. JETP. 1988. V. 67. N 4. P. 714.

[2] Аверкиев Н.С., Дьяконов М.И. // Опт. и спектр. 1990. Т. 68. № 5. C. 1118; Averkiev S., Dyakonov M.I. // Opt. Spectrosc. 1990. V. 68. N 5. C. 653.

[3] Бикеев О.Н., Севастьянов Л.А. // Вестн. РУДН. Сер. МИФ. 2017. Т. 25. № 2. С. 141.

[4] Babicheva V.E., Shalaginov M.Y., Ishii S., Boltasseva A., Kildishev A.V. // Opt. Express. 2015. V. 23. N 24. P. 31109.

[5] Ляшко Е.И., Маймистов А.И. // Квант. электрон. 2015. T. 45. № 11. C. 1050; Lyashko E.I., Maimistov A.I. // Quantum Electronics. 2015. V. 45. N 11. P. 1050. doi 10.1070/QE2015v045n11ABEH015858 
[6] Ляшко Е.И., Маймистов А.И. // Квант. электрон. 2017. T. 47. № 11. С. 1053; Lyashko E.I., Maimistov A.I. // Quantum Electronics. 2017. V. 47. N 11. P. 1053. doi 10.1070/QEL16483

[7] Lyashko E.I., Maimistov A.I. // J. Opt. Soc. Am. B. 2016. V. 33. N 11. P. 2320. doi 10.1364/JOSAB.33.002320

[8] Бикеев О.Н., Ловецкий К.П., Севастьянов А.Л. // Вестн. РУДН. Сер. МИФ. 2018. Т. 26. № 2. С. 119.

[9] Guo Y., Newman W., Cortes C.L., Jacob Z. // Advances in OptoElectronics. 2012. V. 2012. ID 452502. P. 1. doi $10.1155 / 2012 / 452502$

[10] Poddubny A.,Iorsh I., Belov P., Kivshar Yu. // Nat. Photonics. 2013. V. 7. N 12. P. 948. doi 10.1038/nphoton.2013.243

[11] Noginov M., Lapine M., Podolskiy V., Kivshar Yu. // Opt. Express. 2013. V. 21. N 12. P. 14895. doi 10.1364/OE.21.014895

[12] Давидович М.В. // УФН. 2019. Т. 189. № 12 (в печати); Davidovich M.V. // Phys. Usp. 2019. V. 62. N 12 (in press).

[13] Nefedov I.S., Valagiannopoulos C.A., Hashemi S.M., Nefedov E.I. // Sci. Rep. 2013. V. 3. P. 2662. doi 10.1038/srep02662

[14] Козина О.Н., Мельников Л.А. // Изв. Сарат. ун-та. Нов. cep. Сер. Физика. 2019. Т. 19. № 2. С. 122; Kozina O.N., Melnikov L.A. // Izv. Saratov Univ. (N. S.), Ser. Physics. 2019. V. 19. N 2. P. 122 (in Russian). doi 10.18500/1817-30202019-19-2-122-131

[15] Овчаренко А.И., Ермаков О.Е., Song М., Богданов А.A., Иорш И.В., Кившарь Ю.С. // Электроника и микроэлектроника СВЧ. 2015. Т. 1. № 1. С. 54.

[16] Давидович М.В. // ЖЭТФ. 2016. Т. 160. № 6. С. 1069; Davidovich M.V. // JETP. V. 123. N 6. P. 928. doi $10.1134 / \mathrm{S} 106377611611025 \mathrm{X}$

[17] Давидович М.В. // Квант. электрон. 2017. Т. 47. № 6. C. 567; Davidovich M.V. // Quantum Electronics. 2017. V. 47. N 6. P. 567. doi 10.1070/QEL16272

[18] Вайнштейн Л.А. Электромагнитные волны. М.: Радио и связь, 1988. 440 с.

[19] Ландау Л.Д., Лифиии Е.М. Теоретическая физика. Т. 8. Электродинамика сплошных сред. М.: Наука, 1982. 620 с.; Landau L.D., Lifshitz E.M. Electrodynamics of Continuous Media (V. 8 of a Course of Theoretical Physics). Oxford: Pergamon Press, 1960. 474 p.

[20] Zhukovsky V.S., Kidwai O., Sipe J.E. // Opt. Express. 2013. V. 21. N 12. P. 14982.

[21] Давидович М.В. // УФН. 2010. Т. 180. № 6. С. 623; Davidovich M.V. // Phys. Usp. 2010. V. 53. N 6. P. 595. doi 10.3367/UFNr.0180.201006e.0623

[22] Давидович М.В. // Письма в ЖЭТФ. 2018. Т. 108. № 5. C. 228; Davidovich M.V. // JETP Lett. 2018. V. 108. N 5. P. 279. doi 10.1134/S002136401817006X 